\title{
Microfluidic Sub-millisecond Mixers for the Study of Chemical Reaction Kinetics
}

\author{
Amish Desai, Dirk Bökenkamp ${ }^{\dagger}$, Xing Yang, Yu-Chong Tai, Elaine Marzluff ${ }^{\dagger \ddagger}$, Stephen Mayo ${ }^{\dagger \neq}$ \\ Department of Electrical Engineering, Caltech, Pasadena, CA 91125, USA, amish@touch.caltech.edu \\ ${ }^{\dagger}$ Department of Biology, Caltech, Pasadena, CA 91125, USA \\ ${ }^{\sharp}$ Howard Hughes Medical Institute
}

\section{SUMMARY}

We present here, a micromachined, high Reynolds number $(2,000-6,000)$, sub-millisecond liquid mixer for the study of chemical reaction kinetics. This $1 \mathrm{~cm} \times 1 \mathrm{~cm} \times 1 \mathrm{~mm}$ bulk micromachined silicon mixer is capable of initiating and quenching (starting and stopping) chemical reactions in intervals as short as $100 \mu \mathrm{s}$. The centimeter sized mixer chip contains two tee mixers connected by one channel which serves as a reaction chamber. Each tee mixer consists of opposing channels where liquids meet head-on and exit into a third channel forming the base of a "T." Our mixer performance was characterized by employing two carefully chosen chemical reactions with reaction time constants of $3 \mathrm{~ms}$ and $9 \mathrm{~ms}$ along with visualization techniques using dyes and acid-base indicators.

Keywords: mixing, chemical reaction, micro-reactors

\section{INTRODUCTION}

In the study of liquid-phase reaction kinetics, much of the chemistry occurs in sub-millisecond time-scales. For example, many biological processes such as cell activation, enzyme reactions, and protein folding demand sub-millisecond analysis if one wants to investigate intermediate states formed during the reaction. These biochemical processes inevitably involve mixing of certain reactants (i.e. A and B) to initiate the reaction. The schematic below depicts a practical means of investigating intermediate states of a fast liquid-phase reaction. The lines represent fluid channels and the star represent mixing points. As an example, assume A and B react to form product, $\mathrm{C}$.

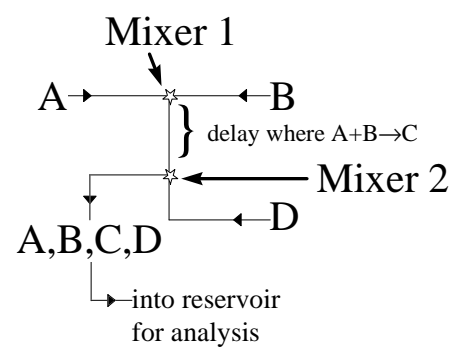

Figure 1: Mixer chip schematic

We would like to able to instantaneously mix liquids A and B together at mixer 1 , control the reaction time by varying the delay (the traveling time between two mixers), and then again be able to instantaneously mix $\mathrm{D}$, a reaction inhibitor, at mixer. (Fig. 1) The collected products can then be collected continuously with a desired volume. The ability to perform this series of high speed mixing steps, therefore, is crucial to the study of chemical reactions which can be analyzed in this fashion. Practically, it is crucial to have fast mixers and short delay (reaction) time if the reaction is fast.

Commercial machines capable of performing this series of fast mixing steps are available today but are limited in several ways. For example, one best available in the market is a series of Berger ball mixers [1] implemented in commercial machines such as the BioLogic SFM4/Q Quenchflow. These larger ball mixers ( 1 in $\times 1 / 2$ in $\times 1 / 2$ in), however, only allow reaction delay times as short as $3 \mathrm{~ms}$. Any significant delay shorter than $3 \mathrm{~ms}$ cannot be studied using these ball mixers because the mixers are not integrated but actually with two separate units. With micromachining, short delay length is obviously available and , more importantly, several mixers can be integrated on one chip and several mixing events in series or parallel can be initiated. The micromachined device also enables smaller dead volumes in the system as well as provides large volumes of samples for examination with continuous operation.

In the field of biochemistry, although chemists like Moskowitz [2] and Takahashi [3] have demonstrated $\mu$ s mixing using multicapillaries and free jet mixing respectively, these methods have very limited use because a second quench cannot be performed after the first jet mixing. The capability of a second quench and, or subsequent mixing steps is key to stop the reaction and thus "freeze" the chemical state of the reactants in time.

In the MEMS field, micro-mixing devices fabricated by Miyake [4] and Schwesinger [5] have primarily operated in low Reynolds numbers regimes resulting in mixing times on the order of seconds. The primary technique has been to increase contact area by interweaving the two fluids. This interweaving has been done by manipulating structural geometry in the channel such as micro-nozzle arrays [4], sinusoidally shaped channels [6], and a divide and mix type method [7]. These MEMS mixers have been characterized primarily with real time visual methods as their time scales allow.

We demonstrate, here, a fundamentally different type of micromachined fast mixer, and demonstrate a different chemical reaction technique to calibrate our mixer. Our results show that the micromachined mixer outperforms the state-of-the-art mixer available today by an order of magnitude.

\section{DESIGN, FABRICATION, \& EXPERIMENTS}

Although the mixing of fluids is fundamentally governed by molecular diffusion, turbulence in the flow can enhance mixing by many orders of magnitude [8]. It is well known that turbulence in the flow field $(\operatorname{Re}>2000)$ will increase reactant area and reduce reaction times. Accordingly, we design our mixers to be in used in the high Reynolds number regime to minimize mixing time. 
We fabricated tee mixers with various delay lengths per chip. The chip and a holder was designed so that they could be exactly substituted for the ball mixer provided with a commercial quenched flow apparatus, BioLogic SFM4/Q (as shown in Fig. 3 ). The semi-vertical, and rough profile of anisotropically etched $<110>$ Si wafers was used to create channels through which high speed fluids could be injected.

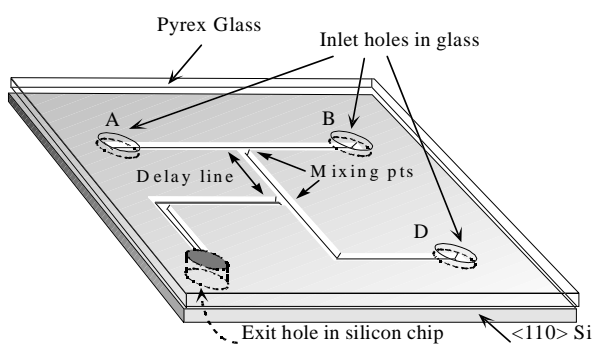

Figure 2: 3-d view of fabricated mixer chip

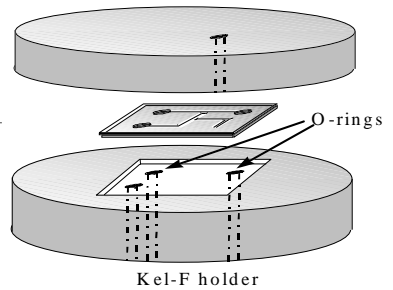

Figure 3: Mixer interface to Biologic machine

The silicon mixer (Fig. 2, 4) is fabricated using <110> $\mathrm{Si}$ wafers, $\mathrm{KOH}$ etching, and anodic glass bonding. Using thermal oxide as a mask, the mixer channels, $150 \mu \mathrm{m}$ deep and $408 \mu \mathrm{m}$ wide, are etched in $70^{\circ} \mathrm{C} \mathrm{KOH}$ for $2.2 \mathrm{hrs}$. An exit hole $(750$ $\mu \mathrm{m}$ diameter) is drilled into the silicon chip before anodically bonding the pyrex 7740 glass, which also has three similarly sized holes. $1 \mathrm{~cm} \times 1 \mathrm{~cm}$ mixer chips with delay lengths of 5.33 $\mathrm{mm}$, and $10.66 \mathrm{~mm}$, respectively, without the glass cover and back holes are shown below in Fig. 4.

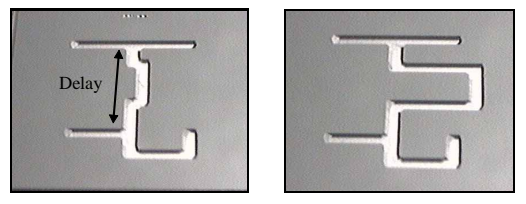

Figure 4: Two mixers with delays $5.33 \mathrm{~mm}$ and $10.66 \mathrm{~mm}$

In order to simplify calibration of the mixer, we designed the mixer chip so that it can be substituted for the BioLogic mixer and use the BioLogic machine to accurately control the input flow rates. The chip is interfaced to the Biologic machine using the kel-f holder and o-rings shown in Fig. 3. Clamping the two holders together seals the chip.

Fluids at precisely controllable flow rates up to $1.5 \mathrm{ml} / \mathrm{s}$ can be injected into the three inlets and ejected from the top. The mechanics of the mixer chip operation are graphically portrayed in Fig. 2. The two fluids to be mixed are first injected in ports A and $\mathrm{B}$, and the two mixing steps occur at the 1st and 2nd mixing point shown. Between these two mixing points, the two fluids are allowed to react in the delay length. Since the dimensions of the channel are known, by controlling the flow rates the reaction time can be controlled. Furthermore, by using a well calibrated chemical reaction, the actual delay between the two mixing steps can be confirmed.

\section{CHEMICAL CALIBRATION METHOD AND THEORY}

The mixer is calibrated with two pseudo 1st order reactions. The first reaction, the hydrolysis of DNPA $(2,4$ dinitrophenylacetate), is a well studied reaction specifically suitable for fast mixing [9]. Mixer calibration relies on the fact that as the DNPA is hydrolyzed by a base, sodium hydroxide, it is converted into DNP (dinitrophenol) (Eq. 1, \& 2).

$$
\begin{aligned}
& -\frac{d C_{D N P A}(t)}{d t} \cong k^{\prime} \cdot C_{D N P A}(t) \\
& \text { where } \mathrm{k}^{\prime}=\mathrm{k} \cdot\left[\mathrm{OH}^{-}\right]
\end{aligned}
$$$$
\mathrm{DNPA}+\mathrm{NaOH} \rightarrow \mathrm{DNP}+\text { Acetic Acid }
$$

$\mathrm{k}$ is the rate constant of this reaction, and $\left[\mathrm{OH}^{-}\right]$represents the hydroxide concentration. This reaction is pseudo 1 st order with a time constant, $\tau=1 / \mathrm{k}^{\prime}$, if $[\mathrm{DNPA}]<<\left[\mathrm{OH}^{-}\right]$, i.e.,

$$
\operatorname{CDNPA}(\mathrm{t})=\operatorname{CDNPA}(0) \cdot e^{-t / \tau}
$$

Experimentally, this reaction is quenched at mixer 2 with $\mathrm{HCl}$ which neutralizes the $\mathrm{NaOH}$. We used mixer chips with different delay times to collect fluids with the corresponding reaction times. Then, the UV absorbance of these fluids is measured to determine concentration of unreacted DNPA. This is possible because the reactant, DNPA, and its reacted product, DNP, have different sensitivities at a wavelength of $320 \mathrm{~nm}$. Mathematically, the absorption dependency to concentration of DNPA and DNP can be described in Eq. 5, where a and b are absorption coefficients of DNPA and DNP respectively.

$$
\left.\mathrm{A}(\mathrm{t})=\mathrm{a} \cdot \mathrm{C}_{\text {DNPA }}(\mathrm{t})+\mathrm{b} \cdot \mathrm{C}_{\text {DNP }}(\mathrm{t})\right)
$$

Combining equations 4 and 5, we can relate the change of absorbance vs. reaction time in a 1 st order exponential function:

$$
\mathrm{A}(\mathrm{t})=\left(\mathrm{A}_{0}-\mathrm{A}_{\infty}\right) \cdot e^{-t / \tau}+\mathrm{A}_{\infty}
$$

\section{RESULTS AND DISCUSSION}

Numerous experiments were done using silicon mixers with reaction delay lengths from $2.66 \mathrm{~mm}$ to $20.66 \mathrm{~mm}$ and flow rates from $100 \mu \mathrm{L} / \mathrm{s}$ to $1000 \mu \mathrm{L} / \mathrm{s}$. By varying the flow rates of DNPA and $\mathrm{NaOH}$ we are able to collect data of absorbance vs. reaction time. Fig. 6 shows an example where $2 \mathrm{M} \mathrm{NaOH}$ has been reacted with DNPA for reaction times of $3 \mathrm{~ms}$ to $12 \mathrm{~ms}$. This base concentration corresponds to a published rate constant of $56 \mathrm{M}^{-1} \mathrm{~s}^{-1}$ [9] and a reaction time constant of $10 \mathrm{~ms}(\tau$, Eq. 4$)$. 


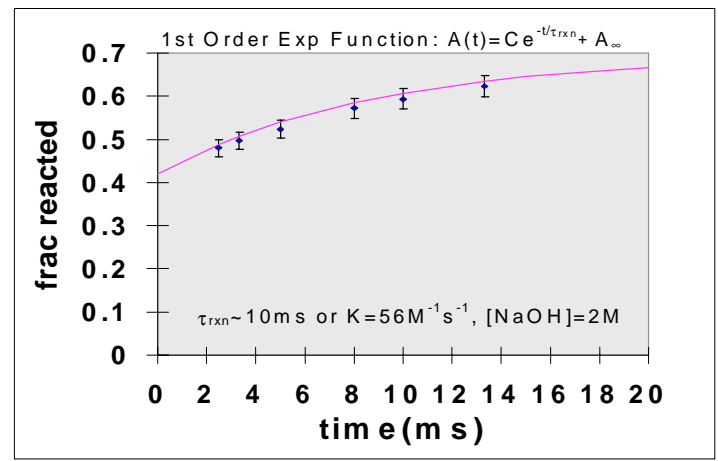

Figure 5: DNPA Absorbance vs. Time Plot

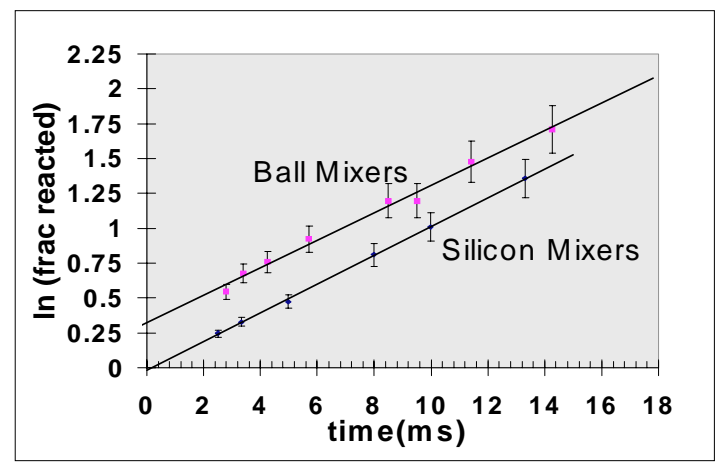

Figure 6: Linearized DNPA data

Figure 6 then shows a series of experiments done with both the commercial and silicon mixers for comparison. Linear fits of these experiments produce rate constants of $51 \mathrm{M}^{-1} \mathrm{~s}^{-1}$ and $46 \mathrm{M}^{-1} \mathrm{~s}^{-1}$ for the silicon and commercial mixers, respectively, which agree well with the published figure of $56 \mathrm{M}^{-1} \mathrm{~s}^{-1}$ for DNPA hydrolysis. The good agreement with the published rate constant illustrates that the reaction delay length on the chip agrees with the 1st order reaction model. This fact is important because it demonstrates mixing time is negligible compared to the reaction delay time. In the ideal case of instantaneous mixing, the linear fits should intersect the origin. In other words, at time zero there should be no products (no change in $A(t))$. Due to the design of the Berger ball mixers, it is likely that there may be some reaction occurring in the mixer stage itself which causes this upward shift of the data. There is also a similar shift associated with the silicon mixers but the value is on the order of the experimental error $(\sim \pm 100 \mu \mathrm{s})$.

As we have designed our mixers to be used in the sub-ms regime, the DNPA experiment could not confirm this good performance. In order to test our mixer further, a faster chemical reaction was necessary. For shorter reaction intervals a reaction with a shorter time constant is required. By looking at Fig. 6, we can see that for sub-millisecond reaction times, the change in absorbance is not significant enough for accurate calibration - the reaction time constant needs to be smaller. Although this modification can be done by simply increasing the base concentration, it has many problems. Firstly, to reduce the reaction time constant to $3 \mathrm{~ms}, 4 \mathrm{M}$ base needs to be used. This high base concentration will result in excess heat which in turn will exponentially vary the reaction rate. Furthermore, $4 \mathrm{M}$ $\mathrm{NaOH}$ has almost twice the viscosity (in comparison with $2 \mathrm{M}$ ) which results in increased back pressure and a lower Re number flow. Lastly, high base concentration is corrosive and will slowly etch the Si channels.

Consequently, a faster reaction, the hydrolysis of PCA (phenylchloroacetate), was chosen with a time constant of $3 \mathrm{~ms}$ using $1 \mathrm{M} \mathrm{NaOH}$. Fourteen experiments were conducted and the rate constant, $430 \pm 47.6 \mathrm{M}^{-1} \mathrm{~s}^{-1}$ with a dead time of $120 \mu \mathrm{s} \pm$ $100 \mu \mathrm{s}$ (measured with the silicon mixers (Fig. 8)) agrees quite well with chemical kinetic theory. More importantly, Fig. 7 reveals the achievement of reaction times as short as $110 \mu \mathrm{s}$. Note that the rate constant derived from silicon mixer PCA experiments differ from the commercial mixer by $20 \%$ $\left(362 \mathrm{M}^{-1} \mathrm{~s}^{-1}\right)$, a similar trend is noted in the DNPA experiments. The commercial mixers consistently seem to predict a rate constant that is $20 \%$ lower from the actual value.

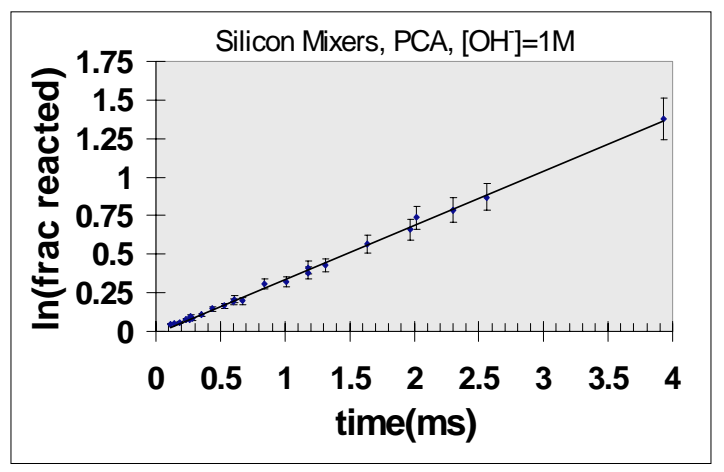

Figure 7: Plot of Silicon Mixers with PCA reaction

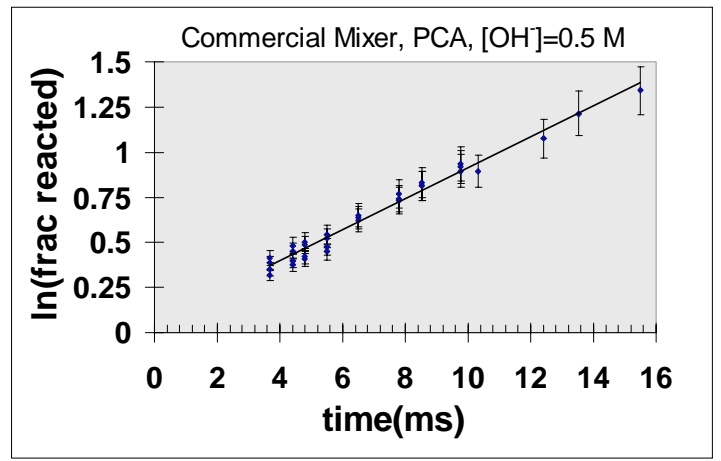

Figure 8: Plot of Commercial Mixers with PCA reaction

It should be emphasized that $10 \%$ to $20 \%$ error in the experimental data is considered very good in these type of quench flow experiments where there are a number of sources of error such as manual pipetting of collected solution, time delay in measurements, and a shift in $\mathrm{NaOH}$ concentrations over time. Nevertheless, one drawback of the PCA reaction is background hydrolysis. The fast PCA reaction is so sensitive that it slowly hydrolyzes by itself. To minimize this background hydrolysis, the PCA solution and its reacted samples used in our experiment are cooled, but it may still contribute errors for some of the shortest reaction time points. Overall, though, the silicon mixers have demonstrated a superb performance which is unavailable from the commercial state-of-the-art instrument. 


\section{FLOW VISUALIZATION}

Here, we then report our work on using flow visualization to confirm the performance of our silicon mixers. In the past, $\mathrm{Li}$ [10] and Bourne [11] have performed flow visualization experiments using acid-base indicators and reaction in millimeter (2-5 $\mathrm{mm}$ diameter) sized pipes to determine downstream mixing distances. To further correlate some of the mixing trends in our silicon mixers, extensive flow visualization experiments were conducted using mixing of dyes and the color change of an acid-base indicator, bromothymol blue. Bromothymol blue remains dark green between $\mathrm{pH}$ 6.0 - 7.6, but turns yellow in the presence of acid and blue in the presence of base. By injecting acid into inlet A (Fig.2), the indicator solution into inlet $\mathrm{B}$, and base into inlet $\mathrm{D}$, three color changes, green to yellow to blue, of the indicator were observed. Photographs of mixer chips with delays lengths of $2.66 \mathrm{~mm}, 5.33 \mathrm{~mm}, 10.66$ $\mathrm{mm}$, and $20.33 \mathrm{~mm}$ while varying flow rates (in the delay channel) from $100 \mu \mathrm{L} / \mathrm{s}$ to $2000 \mu \mathrm{L} / \mathrm{s}$ were taken. Two significant trends were observed. First, at low flow rates, a clearly visible interface between the two streams could be seen; at higher velocities such a boundary could not be discerned. This observation agrees quite well with chemical reaction calibration method. For example, it was noted that consistent reaction data was produced only at flow rates corresponding to flow rates above $\sim 250 \mu \mathrm{L} / \mathrm{s}$. Flow rates below this point resulted in scattered data points below the linear fit. Second, the visualization showed that there were finite mixing delays after the first and second mixing points at all flow rates. These mixing distances, in effect, shifted the actual reaction zone without substantially affecting the reaction delay time for the longer delays. Otherwise, our chemical calibration could not have been successful. In fact, the mixing delays would only contribute significant error for the mixers that have delay lengths of the same order. To illustrate this effect, two pictures of a 2.66 $\mathrm{mm}$ delay chip at $100 \mu \mathrm{L} / \mathrm{s}$ and $500 \mu \mathrm{L} / \mathrm{s}$ are shown below.(Fig. 10) Visual interpretation of these pictures is sketched on the right. The lower photograph shows that 2nd mixing length is almost as large as the reaction pathway itself. To sum up, the flow visualization is an essential component in verifying the validity of the chemical reaction, and should be used in conjuction with the chemical calibration.

\section{CONCLUSION}

As a first demonstration, this project has spawned the fastest sub-millisecond quench flow type mixers available to chemists now. In contrast, the best quench flow mixer commercially available today boasts $\sim 3 \mathrm{~ms}$ as the minimum reaction time. This is a significant leap considering the fact that the best device available today has had little improvement since 1968 [1]. Further optimization of these devices, then, has the possibility of controlling liquid-phase reactions at scales never possible before. Moreover, a chemical reaction technique to calibrate these micromachined mixers has been proven, and verified through flow visualization.

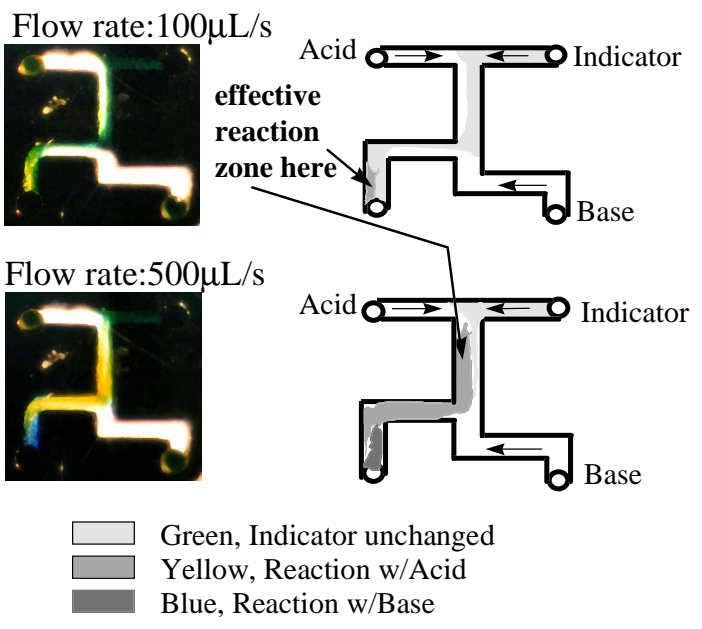

Figure 9: One example of flow visualization

\section{REFERENCES}

1. R. L. Berger, Bohdan Balko, H.F. Chapman, "High Resolution Mixer for the Study of the Kinetics of Rapid Reactions in Solution," Rev. Of Scientific Instr., 1968, V. 39, No. 4.

2. G.W. Moskowitz, R.L. Bowman, "Multicapillary Mixer of Solutions," Science, 153, 1966,428.

3. S. Takahashi, Y. Ching, J. Wang, D.L. Rousseau, "Microsecond Generation of Oxygen_Bound c Oxidase by Rapid Solution Mixing,'J. Of Biological Chemistry, 270,1995, 8405-8407.

4. R. Miyake, T.S. Lammerink, M. Elwenspoek, J. Fluitman, "Micro Mixer with Fast Diffusion," MEMS 1993.

5. N. Schwesinger, T. Frank, "A static micromixer built up in silicon,” SPIE Vol. 2642.

6. Kämper, W. Ehrfeld, J. Döpper, V. Hessel, H. Lehr, H. Lowe, Th. Richter, A. Wolf, "Microfluidic Components for Biological and Chemical Microreactors," MEMS '97.

7. H. Mobius, W. Ehrfeld, V. Hessel, Th. Richter, "Sensor Controlled Processes in Chemical Microreactors," Transducers '95.

8. L. Forney, G. Gray, Optimum Design of a Tee Mixer for Fast Reactions,"AIChE Journal, Nov. 1990, Vol 36.

9. D.J. Cash, G.P. Hess, "Quenched Flow Technique with Plasma Membrane Vesicles: Acetylcholine ReceptorMediated Transmembrane Ion Flux," Anal. Biochem., 112, 39-51, 1981.

10. K. Li, H. Toor, "Chemical Indicators as Mixing Probes. A Possible Way to Measure Micromixing Simply," Ind. Eng. Chem. Fundam. 1986, 25, 719-723.

11. J. Bourne, H. Maire, "Micromixing and Fast Chemical Reactions in Static Mixers," Chem. Eng. Process., 30 (1991) 23-30. 